\title{
Measurement of the residual energy of muons in the Gran Sasso underground Laboratories
}

\author{
The MACRO Collaboration
}

M. Ambrosio ${ }^{12}$, R. Antolini ${ }^{7}$, A. Baldini ${ }^{13}$, G. C. Barbarino ${ }^{12}$, B. C. Barish ${ }^{4}$, G. Battistoni ${ }^{6, b}$, Y. Becherini ${ }^{2}$, R. Bellotti ${ }^{1}$, C. Bemporad ${ }^{13}$, P. Bernardini ${ }^{10}$, H. Bilokon ${ }^{6}$, \%authorC. Bloise ${ }^{6}$, C. Bower ${ }^{8}$, M. Brigida ${ }^{1}$, S. Bussino $^{18}$, F. Cafagna ${ }^{1}$, M. Calicchio ${ }^{1}$, D. Campana ${ }^{12}$, M. Carboni ${ }^{6}$, R. Caruso ${ }^{9}$, S. Cecchini ${ }^{2, c}$, F. Cei ${ }^{13}$, V. Chiarella ${ }^{6}$, B. C. Choudhary ${ }^{4}$, S. Coutu ${ }^{11, i}$, M. Cozzi ${ }^{2}$, G. De Cataldo ${ }^{1}$, H. Dekhissi ${ }^{2,17}$, C. De Marzo ${ }^{1}$, I. De Mitri ${ }^{10}$, J. Derkaoui ${ }^{2,17}$, M. De Vincenzi ${ }^{18}$, A. Di Credico ${ }^{7}$, O. Erriquez ${ }^{1}$, C. Favuzzi ${ }^{1}$, C. Forti ${ }^{6}$, P. Fusco ${ }^{1}$, G. Giacomelli ${ }^{2}$, G. Giannini ${ }^{13, d}$, N. Giglietto ${ }^{1}$, M. Giorgini ${ }^{2}$, M. Grassi ${ }^{13}$, A. Grillo ${ }^{7}$, F. Guarino ${ }^{12}$, C. Gustavino ${ }^{7}$, A. Habig ${ }^{3, p}$, K. Hanson ${ }^{11}$, R. Heinz ${ }^{8}$, E. Iarocci ${ }^{6, e}$, E. Katsavounidis ${ }^{4, q}$, I. Katsavounidis ${ }^{4, r}$, E. Kearns ${ }^{3}$, H. Kim ${ }^{4}$, S. Kyriazopoulou ${ }^{4}$, E. Lamanna ${ }^{14, l}$, C. Lane ${ }^{5}$, D. S. Levin ${ }^{11}$, P. Lipari ${ }^{14}$, N. P. Longley ${ }^{4, h}$, M. J. Longo ${ }^{11}$, F. Loparco ${ }^{1,1}$, G. Mancarella ${ }^{10}$, G. Mandrioli ${ }^{2}$, A. Margiotta ${ }^{2}$, A. Marini ${ }^{6}$, D. Martello ${ }^{10}$, A. Marzari-Chiesa ${ }^{16}$, M. N. Mazziotta ${ }^{1,1}$, D. G. Michael ${ }^{4}$, P. Monacelli ${ }^{9}$, T. Montaruli ${ }^{1}$, M. Monteno ${ }^{16}$, S. Mufson ${ }^{8}$, J. Musser ${ }^{8}$, D. Nicolò ${ }^{13}$, R. Nolty ${ }^{4}$, C. Orth ${ }^{3}$, G. Osteria ${ }^{12}$, O. Palamara ${ }^{7}$, V. Patera ${ }^{6, e}$, L. Patrizii ${ }^{2}$, R. Pazzi ${ }^{13}$, C. W. Peck ${ }^{4}$, L. Perrone ${ }^{10}$, S. Petrera ${ }^{9}$, P. Pistilli ${ }^{18}$, V. Popa ${ }^{2, g}$, A. Rainò ${ }^{1}$, J. Reynoldson ${ }^{7}$, F. Ronga ${ }^{6}$, C. Satriano ${ }^{14, a}$, E. Scapparone ${ }^{7}$, K. Scholberg ${ }^{3, q}$, A. Sciubba ${ }^{6, e}$, P. Serra ${ }^{2}$, M. Sioli ${ }^{2}$, G. Sirri ${ }^{2}$, M. Sitta ${ }^{16, o}$, P. Spinelli ${ }^{1}$, M. Spinetti ${ }^{6}$, M. Spurio ${ }^{2}$, R. Steinberg ${ }^{5}$, J. L. Stone ${ }^{3}$, L. R. Sulak ${ }^{3}$, A. Surdo ${ }^{10}$, G. Tarlè̀ ${ }^{11}$, M. Vakilii ${ }^{15, s}$, C. W. Walter ${ }^{3}$ and R. Webb ${ }^{15}$. 
1. Dipartimento di Fisica dell'Università di Bari and INFN, 70126 Bari, Italy

2. Dipartimento di Fisica dell'Università di Bologna and INFN, 40126 Bologna, Italy

3. Physics Department, Boston University, Boston, MA 02215, USA

4. California Institute of Technology, Pasadena, CA 91125, USA

5. Department of Physics, Drexel University, Philadelphia, PA 19104, USA

6. Laboratori Nazionali di Frascati dell'INFN, 00044 Frascati (Roma), Italy

7. Laboratori Nazionali del Gran Sasso dell'INFN, 67010 Assergi (L'Aquila), Italy

8. Depts. of Physics and of Astronomy, Indiana University, Bloomington, IN 47405, USA

9. Dipartimento di Fisica dell'Università dell'Aquila and INFN, 67100 L'Aquila, Italy

10. Dipartimento di Fisica dell'Università di Lecce and INFN, 73100 Lecce, Italy

11. Department of Physics, University of Michigan, Ann Arbor, MI 48109, USA

12. Dipartimento di Fisica dell'Università di Napoli and INFN, 80125 Napoli, Italy

13. Dipartimento di Fisica dell'Università di Pisa and INFN, 56010 Pisa, Italy

14. Dipartimento di Fisica dell'Università di Roma "La Sapienza" and INFN, 00185 Roma, Italy

15. Physics Department, Texas A\&M University, College Station, TX 77843, USA

16. Dipartimento di Fisica Sperimentale dell'Università di Torino and INFN, 10125 Torino, Italy

17. L.P.T.P, Faculty of Sciences, University Mohamed I, B.P. 524 Oujda, Morocco 18. Dipartimento di Fisica dell'Università di Roma Tre and INFN Sezione Roma

Tre, 00146 Roma, Italy

a Also Università della Basilicata, 85100 Potenza, Italy

$b$ Also INFN Milano, 20133 Milano, Italy

$c$ Also Istituto IASF/CNR, 40129 Bologna, Italy

$d$ Also Università di Trieste and INFN, 34100 Trieste, Italy

e Also Dipartimento di Energetica, Università di Roma, 00185 Roma, Italy

$g$ Also Institute for Space Sciences, 76900 Bucharest, Romania

$h$ Macalester College, Dept. of Physics and Astr., St. Paul, MN 55105

$i$ Also Department of Physics, Pennsylvania State University, University Park, PA 16801, USA

lAlso Dipartimento di Fisica dell'Università della Calabria, Rende (Cosenza), Italy $o$ Also Dipartimento di Scienze e Tecnologie Avanzate, Università del Piemonte

Orientale, Alessandria, Italy

p Also U. Minn. Duluth Physics Dept., Duluth, MN 55812

$q$ Also Dept. of Physics, MIT, Cambridge, MA 02139

$r$ Also Intervideo Inc., Torrance CA 90505 USA

$s$ Also Resonance Photonics, Markham, Ontario, Canada

\section{Abstract}

The MACRO detector was located in the Hall B of the Gran Sasso underground Laboratories under an average rock overburden of $3700 \mathrm{hg} / \mathrm{cm}^{2}$. A transition radiation detector composed of three identical modules, covering a total horizontal 
area of $36 \mathrm{~m}^{2}$, was installed inside the empty upper part of the detector in order to measure the residual energy of muons. This paper presents the measurement of the residual energy of single and double muons crossing the apparatus. Our data show that double muons are more energetic than single ones. This measurement is performed over a standard rock depth range from 3000 to $6500 \mathrm{hg} / \mathrm{cm}^{2}$.

\section{Introduction}

Underground muons are the remnants of the air showers initiated by the collisions of primary cosmic rays with air nuclei. These secondary cosmic ray muons can easily cross large amounts of matter and penetrate into underground laboratories. The energy spectra of underground muons depend on the energy spectra and composition of primary cosmic rays, on their interactions with air nuclei and on the muon energy loss in the rock.

In this paper the measurement of the energy spectra of underground single and double muons, performed with a $36 \mathrm{~m}^{2}$ Transition Radiation Detector (TRD), that was installed in the empty upper part of the MACRO detector [1], is presented. An analysis using part of the single muon data sample was already published [2]. The final sample is approximately 10 times larger and a correct evaluation of the systematics has been performed, thus allowing us to make more reliable conclusions about the spectra and the average residual energy of single and double muon events. The TRD sub-detector, described in sec. 3, uses for triggering purpose and for the measurement of the event multiplicity the larger area $\left(\sim 1000 \mathrm{~m}^{2}\right)$ of the streamer tube and scintillation counters systems. The data selection is described in sec. 4 .

To evaluate the muon energy spectra from the TRD data, we used two complementary approaches described in sec. 6. In the first case, an unfolding procedure was applied, while in the second case a parameterization for the underground muon energy spectrum was assumed and a best fit of the spectral parameters was carried out.

To study how the energy spectra of underground muons are related to the primary cosmic ray spectrum and composition, a dedicated Monte Carlo simulation, described in sec. 7 , was performed. The results of the comparisons between data and Monte Carlo are discussed in sec. 7.1.

1 Corresponding authors: F. Loparco, M. N. Mazziotta Fax: +39080 5442470; e-mail: loparco@ba.infn.it, mazziotta@ba.infn.it 


\section{Underground cosmic ray muons}

The "all-particle" flux of the primary cosmic radiation can be described by an inverse power law energy spectrum [3], with differential flux given by:

$$
\frac{d N}{d E} \propto E^{-(\gamma+1)}
$$

where $\gamma \approx 1.7$ for $E \leq 10^{3} \mathrm{TeV}, \gamma \approx 2.0$ for $10^{3} \mathrm{TeV} \leq E \leq 10^{6} \mathrm{TeV}$ and $\gamma \approx 1.5$ for $E \geq 10^{6} \mathrm{TeV}$.

From the primary spectrum, it is possible to evaluate the energy spectrum at the Earth surface of secondary uncorrelated muons, which is given, with good approximation, by [3]:

$$
\frac{d N_{\mu}}{d \mathcal{E} d \Omega} \approx \text { const } \cdot \mathcal{E}^{-(\gamma+1)} \cdot\left(\frac{1}{1+a \mathcal{E} \cos \theta}+\frac{0.054}{1+b \mathcal{E} \cos \theta}\right)
$$

where $\mathcal{E}$ is the muon energy at the surface, $a=1.1 / 115 \mathrm{GeV}$ and $b=$ $1.1 / 850 \mathrm{GeV}$. The first and the second term in parenthesis of equation 2 represent the contributions of muons from $\pi$ and $\mathrm{K}$ decays, respectively. In the limit of high energies, an approximate expression of the muon surface energy spectrum has the simple form:

$$
\frac{d N_{\mu}}{d \mathcal{E}}=\text { const } \cdot \mathcal{E}^{-\alpha}
$$

where $\alpha=\gamma+2 \approx 3.7$. The surface muon spectral index is therefore related to the primary cosmic ray spectral index.

The energy spectrum of underground muons can be derived taking into account the process of energy loss in the rock, which is assumed to have the form:

$$
\frac{d E}{d h}=-(\lambda+\beta E)
$$

where $d h$ is a thin rock slab (usually in $\mathrm{g} \mathrm{cm}^{-2}$ ), $\lambda$ is the contribution from the ionization energy loss and $\beta E$ is the contribution from radiative processes (bremsstrahlung, pair production and muon hadroproduction). The parameters $\lambda$ and $\beta$ are functions of the muon energy, but for practical purposes can be assumed as constants [3]. The quantity $\epsilon=\lambda / \beta$ is called the critical energy and is defined as the energy value above which the radiative processes become dominant.

With the above assumptions, the general solution of equation 4 is:

$$
E_{\mu}=(\mathcal{E}+\epsilon) e^{-\beta h}-\epsilon .
$$


where $E_{\mu}$ is the muon energy after crossing the rock slant depth $h\left(\mathrm{~g} \mathrm{~cm}^{-2}\right)$. The underground muon energy spectrum can be thus obtained from equations 3 and 5 using the following relation:

$$
\frac{d N}{d E_{\mu}}=\left[\frac{d N}{d \mathcal{E}}\right]_{\mathcal{E}=\mathcal{E}\left(E_{\mu}\right)} \cdot \frac{d \mathcal{E}}{d E_{\mu}}
$$

and is given by:

$$
\frac{d N}{d E_{\mu}}=\text { const } \cdot\left(E_{\mu}+\epsilon\left(1-e^{-\beta h}\right)\right)^{-\alpha} .
$$

From eq. 7, the average underground muon energy at depth $h$ is:

$$
\left\langle E_{\mu}\right\rangle=\frac{\epsilon\left(1-e^{-\beta h}\right)}{\alpha-2}
$$

and its asymptotic value is $\epsilon /(\alpha-2)$. At great depths $h$, the underground muon energy spectrum given by eq. 7 is almost flat for $E \ll\left\langle E_{\mu}\right\rangle$, and then decreases with energy.

\section{The MACRO TRD}

Transition Radiation (TR) is the process of the emission of X-ray photons occurring when an ultrarelativistic charged particle crosses the boundary between two materials with different dielectric constants. The most important features of TR are that the TR yield is roughly proportional to the Lorentz factor $\gamma$ of the radiating particle over a wide range of $\gamma$, and the emission probability of a TR X-ray is of the order of $\alpha \approx 1 / 137$. If the rest mass $m_{0}$ of the radiating particle is known, a measurement of its Lorentz factor $\gamma$ also allows one to evaluate the energy as $E=m_{0} c^{2} \gamma$. TRDs can provide energy measurements over ranges typically spanning one order of magnitude.

Due to the characteristic dependence of the TR yield on the Lorentz factor $\gamma$, TRDs were proposed [4,5] for the measurement of energies of underground cosmic ray muons in the $\mathrm{TeV}$ region. The TRD operated inside the MACRO [1] detector in the Gran Sasso underground Laboratory (LNGS) collected data from April 1995 to June 2000. It was designed to be sensitive to the energy region between $100 \mathrm{GeV}$ and $1 \mathrm{TeV}$.

The detector consisted of three modules covering a total horizontal area of $\sim 36 \mathrm{~m}^{2}$. Each module was composed by eleven $10 \mathrm{~cm}$ thick radiator layers, interleaved by ten planes of 32 proportional tubes $6 \mathrm{~m}$ long and with a square cross section of $6 \times 6 \mathrm{~cm}^{2}$. A detailed description of the detector is given in $[2,5]$. 
The radiator material (Ethafoam 220) contains cells of $\sim 35 \mu \mathrm{m}$ wall thickness and $\sim 900 \mu \mathrm{m}$ spacing, ensuring a threshold Lorentz factor $\gamma_{t h} \approx 10^{3}$ and a saturation Lorentz factor $\gamma_{s a t} \approx 10^{4}$, that correspond to the muon energy range between $\sim 100 \mathrm{GeV}$ and $\sim 1 \mathrm{TeV}$.

The proportional tubes were filled with an $\mathrm{Ar}(90 \%)-\mathrm{CO}_{2}(10 \%)$ gas mixture and were operated at a gain of $\sim 10^{3}$. They were equipped with a cluster counting read-out electronics. Wire signals were sharply differentiated and compared to a threshold corresponding to an energy deposit of $\sim 5 \mathrm{keV}$. In this way it was possible to discriminate $\delta$-ray background from X-ray photoelectrons producing pulses exceeding the threshold amplitude. For each event the pulses with amplitude greater than the threshold ("hits") were counted in all the proportional tubes.

The third TRD module was partially equipped with ten $1 \mathrm{~mm}$ thick aluminum foils of $2 \times 2 \mathrm{~m}^{2}$ area, that were inserted between each radiator and the tube plane below, in the terminal part of the module. The aluminum foils absorbed the TR emitted by muons in the upstream radiator layers. Using this technique a sample of muons were collected with only the ionization loss measured.

A reduced scale prototype of the TRD was exposed to a pion/electron beam [5] to evaluate the detector response function. The physical observable related to the energy of the particles crossing the detector is the total number of hits produced in the proportional tubes. For a sample of particles crossing the detector with a fixed energy and at a fixed angle, the number of hits are roughly Poisson distributed, with an average value of few units, which depends on the beam energy and crossing angle. In Fig. 1 the average number of measured hits in the proportional tubes is plotted as a function of the Lorentz factor $\gamma$ of the incident particles, for several beam crossing angles. Below the threshold value $\left(\gamma=10^{3}\right)$ there is only the contribution of the ionization energy loss; for $10^{3}<\gamma<10^{4}$, the TR contribution is also present and the average number of hits increases logarithmically with $\gamma$.

\section{Data selection and analysis}

We considered the data collected by all the three MACRO TRD modules during the acquisition period from April 1995 to December 2000. Two classes of events were analyzed:

(1) "single muons", i.e. events with one muon in MACRO crossing one of the TRD modules;

(2) "double muons", i.e. events with two muons in MACRO and only one muon crossing one of the TRD modules, like the one shown in figure 2. 
To evaluate the muon energies, we associated to each muon track the hits produced in the TRD proportional tubes. The muon was tracked with the standard MACRO procedure [1], which uses the information of the streamer tube system. The distribution of the distances of the TRD hits from the expected position, calculated with the muon reconstructed track, has a gaussian shape with a standard deviation $\sigma \approx 2 \mathrm{~cm}$. We associated to each muon all the proportional tubes hits within $3 \sigma$ from the track. To avoid badly reconstructed tracks, only muons crossing at least three layers of streamer tubes in the lower part of MACRO were used. Muons accompanied with electromagnetic showers initiated in the rock surrounding the detector were also discarded. Since the TRD was calibrated (see Fig. 1) with particles crossing all the ten layers of proportional tubes at angles below $45^{\circ}$, in the present analysis only muons crossing the whole detector with zenith angle smaller than $45^{\circ}$ were included.

Runs in which the TRD modules were affected by stability problems or malfunctioning were discarded. Also runs with reconstructed muon rates differing more than three standard deviations from the average values were disregarded, as well as those runs whose duration was less than $1 \mathrm{~h}$. The final data samples consist of 250290 single muons and 17942 double muons, for a total life time of 2586 days (see Table 1 ).

Fig. 3 shows the distributions of the number of hits produced in the TRD proportional tubes along the muon tracks for the final data samples. Since the second and third TRD modules were equipped with a different read-out electronics from that of the first module, two different response functions (described in the next section) were necessary to analyze the data samples from the first module and those from the second and third modules. The results of the two analysis were then combined.

\section{The TRD response function}

\subsection{Evaluation of the TRD response function}

We define $N(k \mid h, \theta)$ as the distributions of the TRD hits for a sample of muons with zenith angle $\theta$ that crossed a rock thickness $h$. The rock thickness $h$ was calculated from the direction $(\theta, \phi)$ using the Gran Sasso map [6] and was converted into standard rock according the prescriptions of [7]. These distributions can be related to the residual muon energy spectra $N(E \mid h, \theta)$ 
by:

$$
N(k \mid h, \theta)=\sum_{E} p(k \mid E, \theta) N(E \mid h, \theta)
$$

where $p(k \mid E, \theta)$ is the TR detector response function, i.e. the probability to observe $k$ hits along the track of a muon with underground energy $E=E_{\mu}$, crossing the detector at a zenith angle $\theta$.

To reconstruct the muon energy spectrum $N(E \mid h, \theta)$ starting from the measured hit distributions (Fig. 3), once the TRD response function $p(k \mid E, \theta)$ is known, eq. 9 must be inverted. We derived two matrices $p(k \mid E, \theta)$ (one for the first TR module and another for the second and third modules) on the basis of the calibration data taken by a reduced scale prototype exposed to a pion-electron test beam at CERN [5].

We simulated, with a full GEANT-based [8] Monte Carlo, a muon sample distributed according a flat energy and solid angle spectrum (i.e. $\frac{d^{2} N}{d E d \Omega}=$ constant). On the basis of the calibration data shown in Fig. 1, seven energy bins and four angular bins were defined. The first energy bin covers the range from 0 to $50 \mathrm{GeV}$ (i.e. $0<\gamma<500$ ), and the last one covers the range above the TRD saturation $\left(E \geq 1 \mathrm{TeV}\right.$, i.e. $\left.\gamma \geq 10^{4}\right)$.

The number of hits produced in the TRD by a simulated muon of energy $E$ crossing the detector at zenith angle $\theta$ was extracted by the corresponding calibration data set. The TRD response function (a $31 \times 7 \times 4$ matrix) was calculated as:

$$
p(k \mid E, \theta)=\frac{\mathcal{N}(k \mid E, \theta)}{\mathcal{N}(E, \theta)}
$$

where $\mathcal{N}(k \mid E, \theta)$ is the number of simulated muons with residual energy $E$ and zenith angle $\theta$, producing $k$ hits, with $0 \leq k \leq 30$ and $\mathcal{N}(E, \theta)$ is the total number of simulated muons with energy $E$ crossing the TRD at a zenith angle $\theta$. The simulated data had the same format of experimental data and were processed by the same analysis tools used for real data.

\subsection{Check of the TRD response function}

The accuracy and the time stability of the TRD response function also needed to be taken into account. Although the nominal gas gain of the TRD proportional tubes was the same of the tubes used in the prototype, during the long time-scale data acquisition period their operating conditions were affected by fluctuations. This was due to drifts of some important parameters, like the gas mixture composition, the atmospheric pressure and the temperature. 
A first check of the TRD response function for energies below the threshold for the emission of TR was made using stopping muons, i.e. muons that are absorbed in the lower part of MACRO, that can be easily tagged by imposing simple geometrical cuts. Since the average residual energy of these muons is below a few $\mathrm{GeV}$, we simulated a sample of muons with a flat energy spectrum up to $10 \mathrm{GeV}$. The same algorithm for the selection of stopping muons was applied to both the real and the simulated data samples. The measured hit distributions of stopping muons are well reproduced by the Monte Carlo simulation (see Table 2), thus confirming the reliability of the TRD calibration in the low energy region.

A further check of the detector response function was performed using the data from the part of the third module equipped with aluminum foils. Since the aluminum foils absorbed the TR produced in the upstream radiator, this data sample allows to check the detector response for muons releasing energy in the proportional tubes only by ionization. To take into account the weak dependence of the TRD response on ionization (see Fig. 1), we simulated a sample of muons with the characteristic energy spectrum at MACRO depth (eq. 7) and with the same angular distribution as real data. In Fig. 4 the values of the average number of hits along the muon track versus the muon zenith angle from the Monte Carlo and from the real data are shown. The $\chi^{2} /$ D.o.F. value (evaluated using only statistical errors) is $3.3 / 9$, showing that there is a good agreement between the data and the simulation.

From this analysis we estimated the systematic uncertainty arising from the fluctuations of the detector operating conditions. For each of the four angular bins used for evaluating the TRD response function, we compared the measured hit distributions $N(k \mid \theta)$ with the simulated ones. We remarked that the differences between the experimental average values and the Monte Carlo predictions are within $\pm 5 \%$. For this reason, to take into account the fluctuations of the detector operating conditions, we associated to the average values of each hit distribution of the calibration data set, an uncertainty of $\pm 5 \%$. These uncertainties will propagate to the systematic errors affecting the measurement of the residual energy of muons.

The systematic errors quoted in the present analysis (see sec. 6) are greater than the ones quoted in our previous work [2]. When that analysis was performed, the TRD module equipped with aluminum foils was still not taking data. For this reason, we could estimate the systematic uncertainties only on the basis of the beam test data and we decided to associate to the calibration data set an uncertainty of $\pm 2 \%$, that is lower than the value we adopted for this analysis. 


\section{Evaluation of the muon energy spectra}

As shown in section 5, the residual energy spectra of underground muons are related to the measured hit distributions in the TRD by eq. 9 .

Two different methods were applied to reconstruct the underground muon energy spectra by inversion of eq. 9. The first approach uses the same unfolding procedure described in [2]. In the second approach a parameterization for the muon energy spectrum was assumed and the parameters were derived using a best fit procedure.

\subsection{The unfolding procedure}

Unfolding techniques are widely applied in problems where matrix inversion is required [9]. To reconstruct the energy spectra of single and double muons starting from the hit distributions of Fig. 3, we applied an iterative unfolding procedure $[9,10]$. As an initial energy spectrum (used as a starting point for the unfolding procedure), we assumed eq. 7 for both the single and the double muon events, with parameters: $\alpha=3.7, \beta=0.38310^{-3} \mathrm{hg}^{-1} \mathrm{~cm}^{2}$ and $\epsilon=620 \mathrm{GeV}$. The final reconstructed spectrum does not depend on the choice of the initial spectrum. It only affects the time needed for the procedure to converge $[9,10]$.

The energy spectrum reconstructed after each iteration is used as input for the next iteration. At the end of each iteration a $\chi^{2}$ test is performed between the reconstructed energy spectra and the input energy spectra. The $\chi^{2}$ is defined as:

$$
\chi^{2}=\sum_{i, j, k} \frac{\left[N_{n}\left(E_{i} \mid h_{j}, \theta_{k}\right)-N_{n-1}\left(E_{i} \mid h_{j}, \theta_{k}\right)\right]^{2}}{\sigma_{n}^{2}\left(E_{i} \mid h_{j}, \theta_{k}\right)+\sigma_{n-1}^{2}\left(E_{i} \mid h_{j}, \theta_{k}\right)}
$$

where $N_{n-1}\left(E_{i} \mid h_{j}, \theta_{k}\right)$ and $N_{n}\left(E_{i} \mid h_{j}, \theta_{k}\right)$ are the energy spectra reconstructed after the $(n-1)$-th and the $n$-th iteration and $\sigma_{n-1}\left(E_{i} \mid h_{j}, \theta_{k}\right)$, while $\sigma_{n}\left(E_{i} \mid h_{j}, \theta_{k}\right)$ are the associated errors. The iterative procedure stops when the energy distribution reconstructed after the $n^{\text {th }}$ iteration has a $\chi^{2}$ probability greater than $99 \%$ to be equivalent to the one reconstructed after the $(n-1)^{t h}$ iteration. The unfolding procedure was separately applied to the data samples from the first and from the second and third TRD modules and the results were combined.

Fig. 5 shows the reconstructed differential energy spectra of single and double muons at the Gran Sasso underground laboratory depth. The error bars in the figure were calculated by adding in quadrature statistical and 
systematic errors. The systematic errors are originated by fluctuations of the TRD response function, and were estimated by a $+5 \%$ and a $-5 \%$ variation of the calibration data. The unfolding procedure was replicated using the "perturbed" detector response functions; the systematic uncertainty was evaluated as the difference between the two results.

The unfolding procedure reconstructs the shapes of the spectra up to $1 \mathrm{TeV}$. For energies greater than $1 \mathrm{TeV}$, where the TRD response is saturated, the spectral shapes cannot be reconstructed, and only the number of events can be evaluated. The average value of the energy of underground muons with energy above threshold, $\langle E\rangle_{E>E_{0}}$, was calculated from eq. 7 as:

$$
\langle E\rangle_{E>E_{0}}=\frac{\alpha-1}{\alpha-2} \cdot\left(E_{0}+\frac{\epsilon\left(1-e^{-\beta h}\right)}{\alpha-1}\right) .
$$

The average muon energy was evaluated as:

$$
\langle E\rangle=(1-f)\langle E\rangle_{E<E_{0}}+f\langle E\rangle_{E>E_{0}}
$$

where $\langle E\rangle_{E<E_{0}}$ is the average energy of muons with energies up to $E_{0}=1 \mathrm{TeV}$, $f$ and $\langle E\rangle_{E>E_{0}}$ are the fraction and the average energy of muons with energies greater than $1 \mathrm{TeV}$, respectively. For a $3 \%$ variation of the parameters $\alpha, \beta$ and $\epsilon$, as is typically quoted by various authors (e.g. [11]), the uncertainties on the average muon energies are less than $1 \%$ and are significantly smaller than our quoted errors. The results are shown in Table 3. The average energy of single muons and double muons are respectively of $270 \pm 3$ (stat.) \pm 18 (syst.) GeV and $381 \pm 13$ (stat.) \pm 21 (syst.) GeV. The single muon result is not in contradiction with the result of our previous analysis [2], where the systematic effects were underestimated.

\subsection{Best fit of the spectral indices}

An alternative way for evaluating the muon energy spectra from the measured hit distributions is that of assuming eq. 7 as an analytic description for the spectra, and to derive the parameters using a best-fit. Substituting the trial energy spectrum into eq. 9 and summing over the zenith angles we get:

$$
N(k \mid h)=\sum_{E} \sum_{\theta} p(k \mid E, \theta) c(h, \theta)\left[E+\epsilon\left(1-e^{-\beta h}\right)\right]^{-\alpha}
$$

where the normalization constants $c(h, \theta)$ represent the number of muons detected in each bin of depth and zenith angle. We fixed the values of the $\beta$ and $\epsilon$ parameters to $\beta=0.38310^{-3} \mathrm{hg}^{-1} \mathrm{~cm}^{2}$ and $\epsilon=620 \mathrm{GeV}$ [12]; $\alpha$ was left as the free parameter for the single and double muon data. 
For each value of $\alpha$ in the range from 2 to 6 and step 0.1 we built a set of hit distributions $\hat{N}(k \mid h)$ according eq. 14, and for each set of distributions we evaluated the $\chi^{2}$ as:

$$
\chi^{2}=\sum_{T R D \text { modules }} \sum_{h} \sum_{k} \frac{[N(k \mid h)-\hat{N}(k \mid h)]^{2}}{\sigma^{2}(k \mid h)+\hat{\sigma}^{2}(k \mid h)}
$$

where $N(k \mid h)$ is the measured hit distribution for muons (single or double) crossing a rock slant depth $h ; \sigma(k \mid h)$ and $\hat{\sigma}(k \mid h)$ are the Poissonian errors on $N(k \mid h)$ and $\hat{N}(k \mid h)$, respectively.

The curves representing the $\chi^{2}$ as a function of $\alpha$ are continuous both for single and double muons and show a well defined minimum. The value of the best fit spectral index for single muons is $\alpha_{1}=3.79 \pm 0.02$ (stat) \pm 0.11 (syst), with $\chi_{\min }^{2} /$ d.o.f. $=1.51$, while for the double muons is $\alpha_{2}=3.25 \pm 0.06$ (stat) \pm 0.07 (syst), with $\chi_{\text {min }}^{2} /$ d.o.f. $=0.55$. The statistical error on $\alpha$ was evaluated using the values $\alpha_{L}$ and $\alpha_{R}$ corresponding to $\chi^{2}=\chi_{\min }^{2}+1$. The systematic error was estimated from the positions of the new minima of $\alpha$ obtained using the two TRD response functions evaluated from the sets of "perturbed" calibration data.

The result obtained for the single muon spectral index is consistent with that obtained from the MACRO measurement of the underground muon intensity as a function of the rock depth [13]. It is also consistent with the results of the NUSEX experiment [14], that found a spectral index of $\alpha=3.91_{-0.36}^{+0.50}$ for a sample of events mainly composed by single muons.

\subsection{Comparison between the results}

Fig. 6 shows a comparison between the differential muon energy spectra reconstructed with the two methods described in sections 6.1 and 6.2 both for single and double muons. In the case of single muons we find a value of $\chi^{2} /$ d.o.f. $=1.3 / 7$, while in the case of double muons we find $\chi^{2} /$ d.o.f. $=8.5 / 7$. In the case of single muon data, there is a good agreement between the result obtained using the unfolding procedure and that obtained using the best fit method. In the case of double muon data, there are some discrepancies, especially for low residual energies.

To understand these difference, it should be noted that the result of the unfolding procedure does not depend on the trial spectrum. The spectrum used for the fit (eq. 7) is derived assuming that the surface muon energy spectra obey a power law with spectral index $\alpha$, and in the muon propagation formula, $\epsilon$ and $\beta$ are constant, i.e. the cross sections for the radiative processes do not depend on the muon energy. 
If the correct expression (eq. 2) is assumed for the surface energy spectrum and the dependence of $\epsilon$ and $\beta$ on the muon energy is taken into account, it is impossible to obtain an analytical expression for the underground muon spectrum. Hence, eq. 7 represents only a useful parameterization for the muon underground spectra, whose accuracy is limited by the hypotheses from which it has been derived. The difference between our results obtained using the two techniques mentioned in sections 6.1 and 6.2 are ascribed to these approximations. We can conclude that the parameterization is not completely suitable for describing the underground muon energy spectra, especially for events with large underground multiplicity.

\section{Monte Carlo simulation}

As shown in section 2, the underground muon energy spectrum is related to the primary cosmic ray spectrum. In order to investigate the relationship between the underground muon energy and the primary cosmic ray spectrum and composition, we performed a Monte Carlo simulation using different composition models for primary cosmic rays.

The interactions of cosmic rays in the atmosphere were simulated with the HEMAS code [15], while the process of muon propagation in the rock was simulated using the PROPMU code [12]. Two extreme composition models were assumed for primary cosmic rays:

a. the "light model" [16], i.e. a proton-rich composition model;

b. the "heavy model" [17], i.e. a Fe-rich composition model.

These models assume that cosmic rays are composed by five main mass groups $(p, H e, C N O, M g$ and $F e)$. The energy spectrum of each component is described by means of power laws given by:

$$
\frac{d N_{i}}{d E}=\left\{\begin{array}{l}
K_{1} E^{-\left(\gamma_{1}+1\right)} E \leq E_{c u t} \\
K_{2} E^{-\left(\gamma_{2}+1\right)} E>E_{c u t}
\end{array}\right.
$$

In eq. 16 there are 5 parameters for each primary component (i.e. the normalization constants $K_{1}$ and $K_{2}$, the spectral indices $\gamma_{1}$ and $\gamma_{2}$ and the cutoff energy $E_{\text {cut }}$ ). These parameters are not independent: usually the constant $K_{2}$ is expressed as a function of the others by imposing the continuity of the function $d N_{i} / d E$ at $E=E_{c u t}$. The all-particle spectrum is evaluated by adding the contributions from all the mass groups. In Table 4 the values of

all the parameters are summarized for each component of the primary cosmic 
rays in both the composition models we adopted. The light model is different from the heavy model because there is an extra-component of protons.

Fig. 7 shows the predictions obtained using the light and heavy composition models for the average energy of the underground muons as a function of the muon multiplicity. The gap between the predictions from the two extreme models increases with the muon multiplicity. The two dark points represent our measured values, averaged on the whole rock depth range from 3000 to $6500 \mathrm{hg} / \mathrm{cm}^{2}$.

To explain the average energy behavior, in Fig. 8 we plotted, for the two composition models and as a function of the underground muon multiplicity, for the $p, H e$ and $F e$ components: $a$ ) the average energies of underground muons; $b$ ) the relative contribution of these three mass groups and $c$ ) the average energies of the parent cosmic rays to events for a fixed underground muon multiplicity. In $a$ ) and $c$ ) the values for the all-particle spectrum are also shown. From this figure the following conclusions can be drawn:

a. single muons are originated mainly by primary protons;

b. the contributions of primary heavy elements become relevant when the muon multiplicities increase, while the contribution of protons decreases;

c. in the "heavy" composition model the contribution of $F e$ nuclei is relevant from low multiplicities (it is $\sim 15 \%$ for $N_{\mu}=2$ and tends to $100 \%$ for high multiplicities);

d. in the light model, the main contribution is always that of protons;

e. for a fixed muon multiplicity, muons originated by light primaries are more energetic than muons originated by heavy primaries.

From the simulation we get that the average energies of underground muons produced by each component are almost independent on the primary composition model. This is due to the fact that the differences between the spectral indices of the primary components in the models we are examining are small for energies below the "knee", as can be seen from table 4. As a consequence, the muons produced e.g. by He nuclei have the same average energy, independently on the choice of the composition model. Hence, the differences between the predictions of the cosmic ray composition models are mainly due to differences in the weights of the various components in each model and not to differences in their spectra.

From Fig. $8 c$, the primary energy needed to produce one underground muon at the MACRO depth is smaller than $100 \mathrm{TeV}$, while the primary energy needed to produce two underground muons is about $300-400 \mathrm{TeV}$. This means that an analysis of events with only one or two underground muons is sensitive to the energy region below the knee of the primary spectrum.

Our measurements were compared with the predictions from the simulation. 
Fig. 7 shows a comparison between the results obtained applying the the analysis technique described in sec. 6.1 and the Monte Carlo predictions concerning the average energies of single and double muons, while in Fig. 9 the average energies of single and double muons are plotted as a function of the rock thickness crossed by muons.

\subsection{Discussion of the results}

Single muon data are not in contradiction with the predictions from both the composition models. In fact, since the energy gaps between the predictions from the two extreme composition models are of the same order of magnitude as the error bars, single muons do not allow one to discriminate between different cosmic ray composition models. The sensitivity of this measurement to the primary cosmic ray composition is strongly limited by systematic errors associated to the TRD response function.

Also in the case of double muons, our data do not allow to perform a discrimination between the cosmic ray composition models. In this case, although the errors associated to the average muon energies are smaller than the gap between the predictions from the two extreme composition models, experimental data do not provide a clean signature in favour of a given composition model.

Another comparison between the TRD data and the Monte Carlo predictions can be done by looking at the spectral indices. For each composition model we fitted the underground energy spectra of single and double muons with the formula 7 , assuming the same values of $\epsilon$ and $\beta$ as in sec. 6.2 and we compared the fit results with the TRD data. Table 5 shows the comparison of the data with the Monte Carlo predictions. As in the previous case, the single and double muon spectral indices do not allow to perform a study of the cosmic ray composition.

The sensitivity of our measurement to the primary cosmic ray composition is mainly limited by its precision and by the poor statistics of the high multiplicity muon events, that does not allow to reconstruct their spectra. A detector with a larger area than the MACRO TRD and with a reduced systematics could enhance the precision of the measurement of the single and double muon energies and also allow to measure the energies of high multiplicity muons, that are more sensitive to the cosmic ray composition in the energy region of the knee. 


\section{Conclusions}

The MACRO TRD allowed the measurement of the energies of muons penetrating in the Gran Sasso underground laboratories, in the standard rock depth range from 3000 to $6500 \mathrm{hg} / \mathrm{cm}^{2}$. For reconstructing the muon energy spectra we used an unfolding method and a best fit procedure. The average energies of single and double muons, evaluated with the unfolding technique described in sec. 6.1, are $\langle E\rangle_{1}=270 \pm 3$ (stat) \pm 18 (syst) GeV and $\langle E\rangle_{2}=381 \pm 13$ (stat) \pm 21 (syst) $\mathrm{GeV}$. The spectral indices for the approximate parameterization of the muon energy spectra (eq. 7), evaluated with the best fit procedure described in sec. 6.2 are $\alpha_{1}=3.79 \pm 0.02$ (stat) \pm 0.11 (syst) and $\alpha_{2}=3.25 \pm 0.06($ stat $) \pm 0.07$ (syst).

We also performed a Monte Carlo simulation to study how the muon energy spectra depend on the primary cosmic ray energy spectra and composition, on their interactions with the atmosphere and on the muon propagation in the rock. Our data show that double muons are more energetic than single ones in the standard rock depth range from 3000 to $6500 \mathrm{hg} / \mathrm{cm}^{2}$, as predicted by our Monte Carlo simulation. The Monte Carlo simulation also showed that a measurement of the energies of underground high multiplicity muons could provide useful information about the primary cosmic ray composition. In fact, we remarked that the differences between the predictions from the various composition models become relevant at high muon multiplicities, where the contribution from heavy primaries is more significant.

The energy spectra of single and double muons reconstructed from our data are in agreement with the Monte Carlo predictions obtained assuming two extreme cosmic ray composition models. This measurement do not allow to perform a cosmic ray composition study because the errors are compatible with the gaps between the predictions from the two models. However, a measurement of the energy spectra of high multiplicity underground muons could provide a useful tool for investigating the primary cosmic ray composition in the energy region above the knee of the spectrum.

\section{References}

[1] S. Ahlen et al.[The MACRO Collaboration], Nucl. Instrum. Methods A 324, 337 (1993), M. Ambrosio et al.[The MACRO Collaboration], accepted for publication by Nucl. Instrum. Methods A.

[2] M. Ambrosio et al.[The MACRO Collaboration], Astrop. Phys. 10, 11 (1999).

[3] T. K. Gaisser, Cosmic Rays and Particle Physics, Cambridge University Press (1990). 
[4] R. Bellotti et al., Nucl. Instrum. Methods A 305, 192 (1991).

[5] E. Barbarito et al., Nucl. Instrum. Methods A 365, 214 (1995).

[6] M. Ambrosio et al.[The MACRO Collaboration], Phys. Rev. D 52, 2673 (1995).

[7] A. G. Wright, Proc. ot the XII ICRC, Denver (USA), 3 (1973), 1709.

[8] R. Brun et al., CERN Publication DD/EE/84-1 (1992).

[9] G. D'Agostini, Nucl. Instrum. Methods A 362, 487 (1995).

[10] M. N. Mazziotta, LNGS 52/95 (July 1995)

[11] G. Battistoni, Nucl. Instrum. Methods A 394, 136 (1997).

[12] P. Lipari, T. Stanev, Phys. Rev. D 44, 3543 (1991).

[13] M. Ambrosio et al., Phys. Rev. D 52, 3793 (1995).

[14] C. Castagnoli et al., Astrop. Phys. 6, 187 (1997).

[15] C. Forti et al., Phys. Rev. D 42, 3668 (1990).

[16] C. Fichtel and J. Linsley, Astroph. J. 300, 474 (1986).

[17] J. Goodman et al., Phys. Rev. D 26, 1043 (1982). 


\begin{tabular}{||l|c|c|c|c||}
\hline \hline & Module 1 & Module 2 & Module 3 & TRD \\
\hline \hline Analyzed runs & 6134 & 3261 & 4218 & \\
Bad runs & 3538 & 2160 & 1897 & \\
Live time & $29004 \mathrm{~h}$ & $14942 \mathrm{~h}$ & $18122 \mathrm{~h}$ & $62069 \mathrm{~h}$ \\
\hline Single $\mu$ after cuts & 128903 & 66675 & 54712 & 250290 \\
\hline Double $\mu$ after cuts & 8904 & 4576 & 4463 & 17943 \\
\hline \hline
\end{tabular}

Table 1

Summary of the data collected by the MACRO TRD. Third module data refers only to the part of the module not equipped with aluminum foils.

\begin{tabular}{|l|c|c||c|c||}
\hline \hline & \multicolumn{2}{|c||}{ TRD DATA } & \multicolumn{2}{c||}{ MC SIMULATION } \\
\cline { 2 - 5 } & $\begin{array}{c}\text { Number of } \\
\text { events }\end{array}$ & $\begin{array}{c}\text { Average } \\
\text { number of hits }\end{array}$ & $\begin{array}{c}\text { Number of } \\
\text { events }\end{array}$ & $\begin{array}{c}\text { Average } \\
\text { number of hits }\end{array}$ \\
\hline \hline Module 1 & 223 & $2.06 \pm 0.12$ & 3273 & $2.12 \pm 0.03$ \\
Modules 2+3 & 283 & $2.05 \pm 0.13$ & 3273 & $2.07 \pm 0.04$ \\
\hline \hline
\end{tabular}

Table 2

Stopping muon data: the measured hit distributions are compared with the predictions from the simulation.

\begin{tabular}{|l|c|c|c|}
\hline \hline & $\begin{array}{c}\langle\mathbf{E}\rangle_{\mathbf{E}<\mathbf{E}_{\mathbf{0}}} \\
(\mathbf{G e V})\end{array}$ & $\mathbf{f}$ & $\langle\mathbf{E}\rangle$ \\
& $\%$ & $(\mathbf{G e V})$ \\
\hline Single muons & $195 \pm 2_{\text {sta }} \pm 15_{\text {sys }}$ & $4.5 \pm 0.1_{\text {sta }} \pm 0.7_{\text {sys }}$ & $270 \pm 3_{\text {sta }} \pm 18_{\text {sys }}$ \\
Double muons & $234 \pm 11_{\text {sta }} \pm 18_{\text {sys }}$ & $9.0 \pm 0.5_{\text {sta }} \pm 1.0_{\text {sys }}$ & $381 \pm 13_{\text {sta }} \pm 21_{\text {sys }}$ \\
\hline \hline
\end{tabular}

Table 3

Average energy of underground single and double muons, with residual energy below the saturation threshold $\left(E_{0}=1 \mathrm{TeV}\right)$ of our TRD (column 2); fraction of events with energy above the threshold (column 3); average energy of all events below and above threshold (column 4). 
Light model

\begin{tabular}{||l|c|c|c|c||}
\hline Group & $K_{1}\left(\mathrm{~m}^{-2} \mathrm{~s}^{-1} \mathrm{sr}^{-1} \mathrm{GeV}^{\gamma_{1}}\right)$ & $\gamma_{1}$ & $E_{\text {cut }}(\mathrm{GeV})$ & $\gamma_{2}$ \\
\hline $\mathrm{p}$ & $1.5 \times 10^{4}$ & 1.71 & $2.0 \times 10^{4}$ & \\
$\mathrm{He}$ & $1.87 \times 10^{3}$ & 1.50 & $3.0 \times 10^{6}$ & 2.0 \\
$\mathrm{CNO}$ & $5.69 \times 10^{3}$ & 1.71 & $3.0 \times 10^{6}$ & 2.0 \\
$\mathrm{Mg}$ & $3.30 \times 10^{3}$ & 1.71 & $3.0 \times 10^{6}$ & 2.0 \\
$\mathrm{Fe}$ & $2.60 \times 10^{3}$ & 1.71 & $3.0 \times 10^{6}$ & 2.0 \\
\hline \multicolumn{4}{|l}{} \\
\hline
\end{tabular}

Heavy model

\begin{tabular}{||l|c|c|c|c||}
\hline Group & $K_{1}\left(\mathrm{~m}^{-2} \mathrm{~s}^{-1} \mathrm{sr}^{-1} \mathrm{GeV}^{\gamma_{1}}\right)$ & $\gamma_{1}$ & $E_{\text {cut }}(\mathrm{GeV})$ & $\gamma_{2}$ \\
\hline $\mathrm{p}$ & $1.5 \times 10^{4}$ & 1.71 & $1.0 \times 10^{5}$ & 2.0 \\
$\mathrm{He}$ & $5.69 \times 10^{3}$ & 1.71 & $2.0 \times 10^{5}$ & 2.0 \\
$\mathrm{CNO}$ & $3.30 \times 10^{3}$ & 1.71 & $7.0 \times 10^{5}$ & 2.0 \\
$\mathrm{Mg}$ & $2.60 \times 10^{3}$ & 1.71 & $1.2 \times 10^{6}$ & 2.0 \\
$\mathrm{Fe}$ & $3.48 \times 10^{3}$ & 1.36 & $2.7 \times 10^{6}$ & 2.0 \\
\hline
\end{tabular}

Table 4

Parameters of the primary cosmic ray energy spectra according the "light" and "heavy" composition models.

\begin{tabular}{||c||c|c||c|c||}
\hline \hline \multicolumn{1}{||c||}{} & \multicolumn{2}{c||}{ Single muons } & \multicolumn{2}{c||}{ Double muons } \\
\cline { 2 - 5 } & $\alpha$ & $\chi^{2} /$ d.o.f. & $\alpha$ & $\chi^{2} /$ d.o.f. \\
\hline TRD data & $3.79 \pm 0.02 \pm 0.11$ & 1.51 & $3.25 \pm 0.06 \pm 0.07$ & 0.55 \\
\hline Light model & 3.70 & 1.55 & 3.14 & 0.92 \\
Heavy model & 3.84 & 1.73 & 3.36 & 1.02 \\
\hline \hline
\end{tabular}

Table 5

Results of the fits of underground muon energy spectra with the formula 7. TRD data (with the statistical and systematic error) are compared with the Monte Carlo predictions obtained assuming two different composition models for primary cosmic rays. 


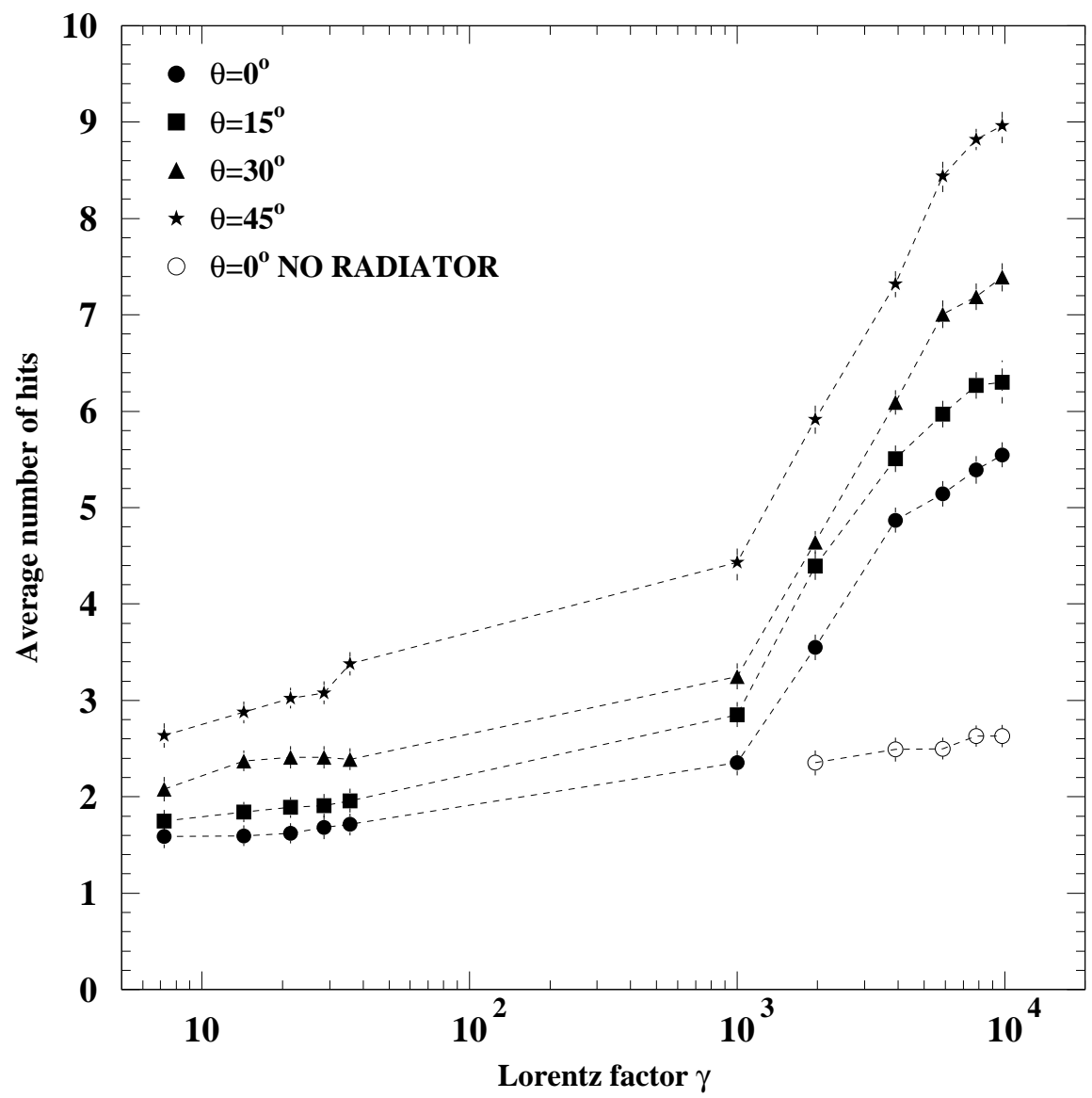

Figure 1. Average number of hits as a function of the Lorentz factor $\gamma$ for several beam crossing angles. The dashed lines are drawn to guide the eye. 

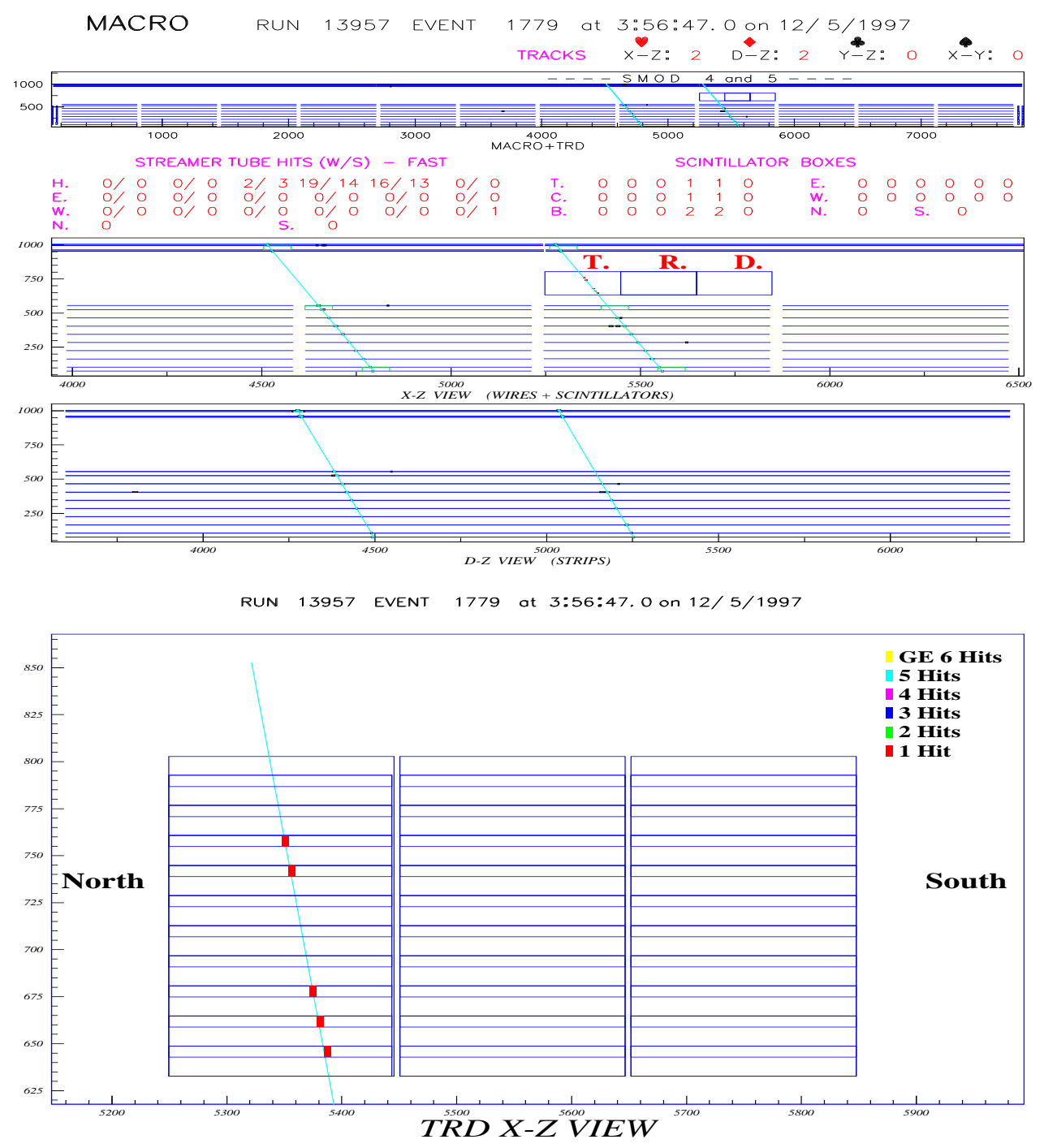

Figure 2. Display of a double muon event. 


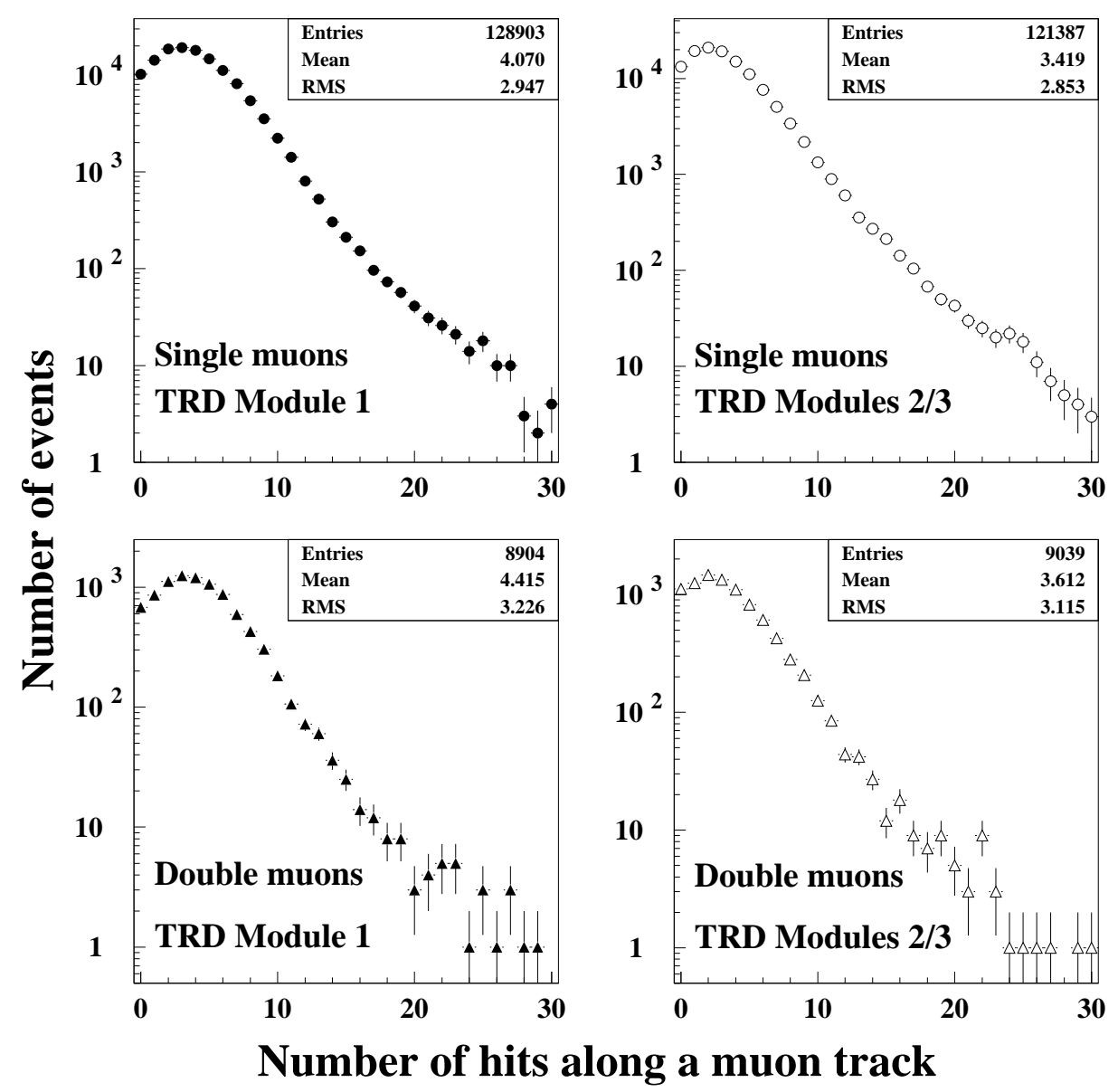

Figure 3. Hit distributions $N(k)$ vs. $k$ for the final single and double muon data samples. Since the read-out electronics of the first TRD module was different from that of the second and third modules, the data data samples from the first module and the ones from the second and third modules were analyzed separately. 


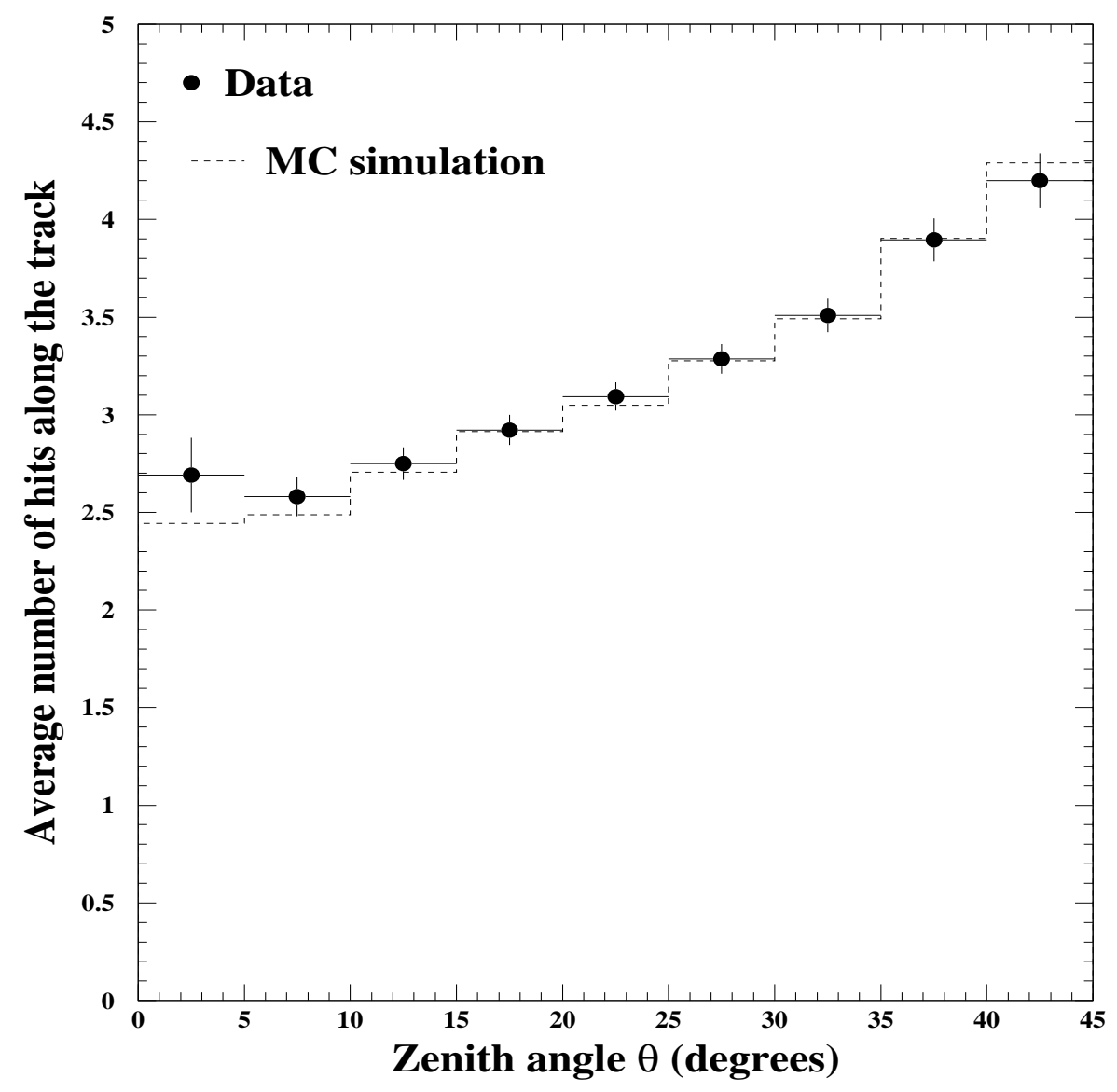

Figure 4. Check of the TRD response function by means of muons crossing the region of the third module equipped with aluminum foils. Data $(\bullet)$ are compared with the Monte Carlo predictions(dashed line). 


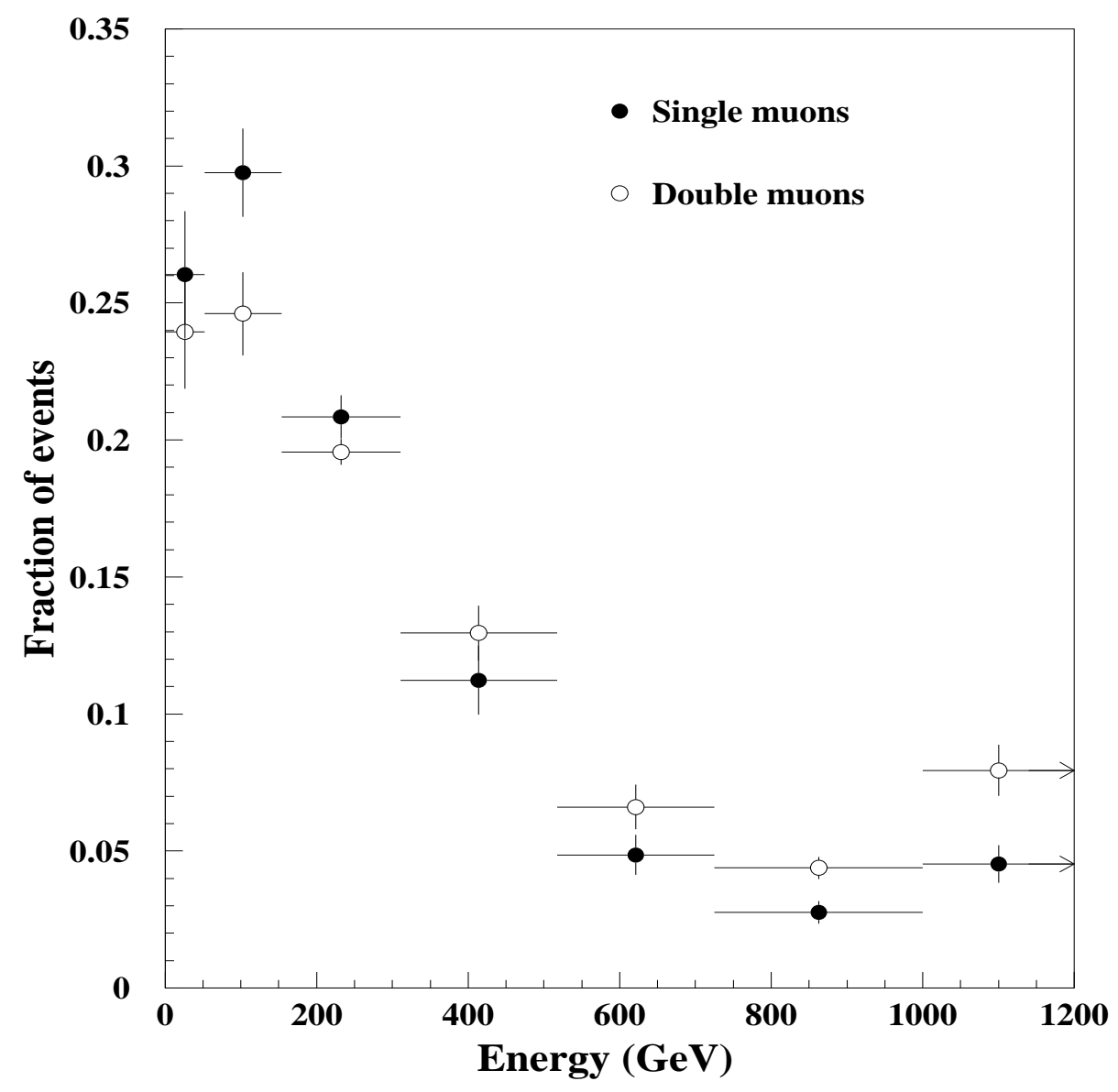

Figure 5. Reconstructed differential energy spectra of single and double muons. Statistical and systematic errors have been added in quadrature. 


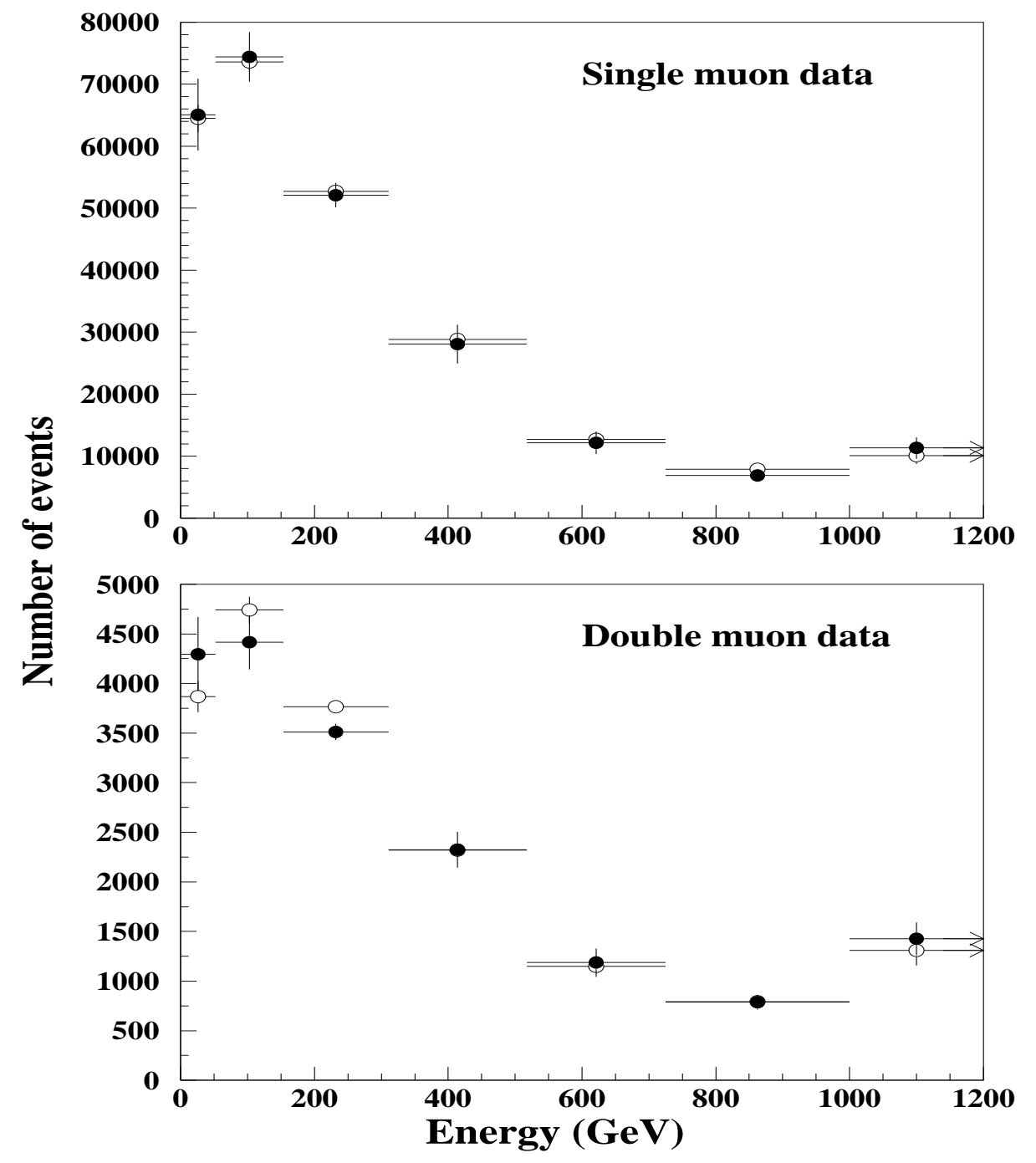

Figure 6. Reconstructed differential energy spectra of single and double muons. Results from the unfolding procedure (black dots) are compared with those from the best fit procedure (open circles). Statistical and systematic errors have been added in quadrature. 


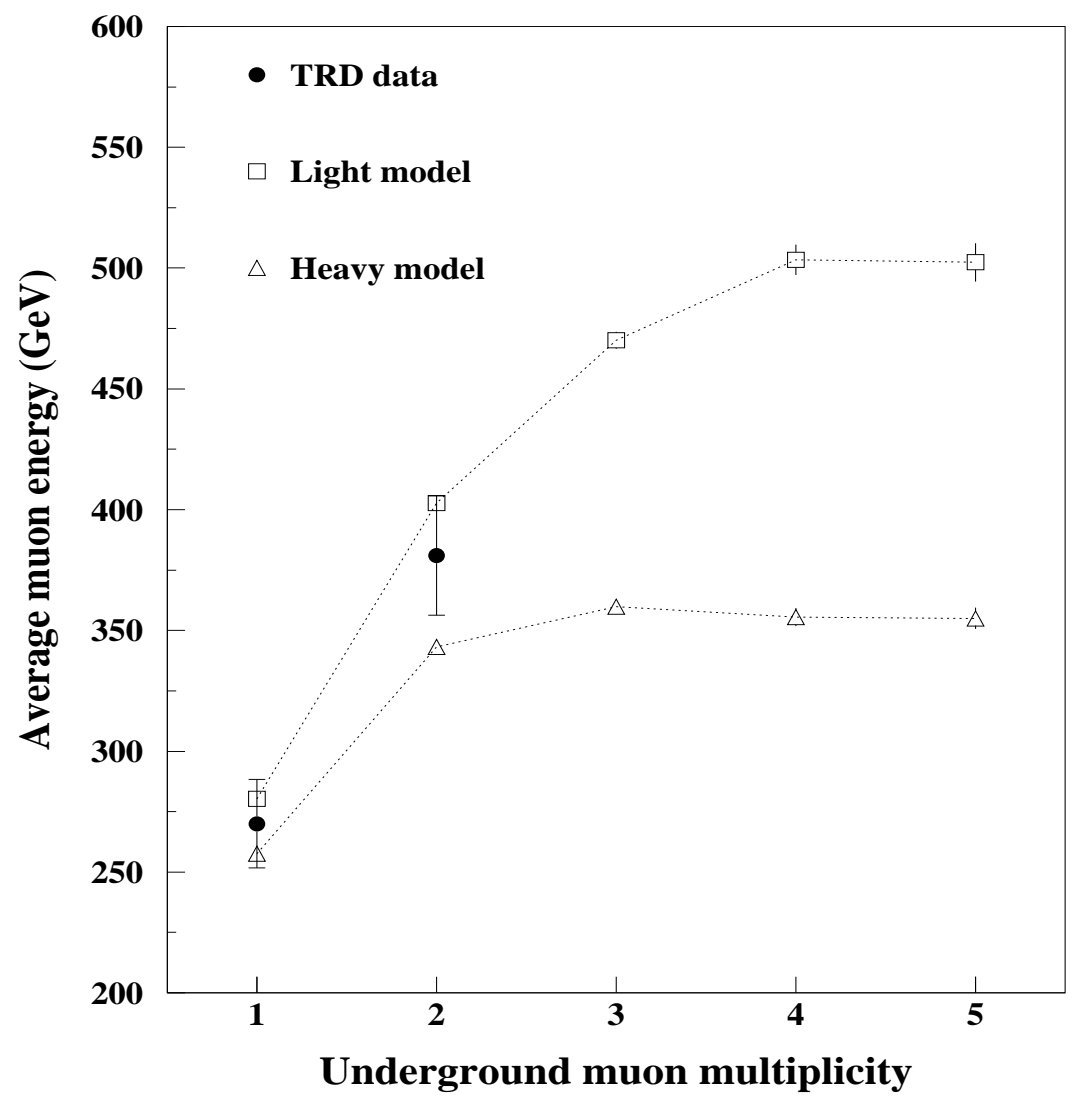

Figure 7. Average energies of underground muons as a fuction of their multiplicity according the predictions obtained assuming the light and heavy composition models. The Monte Carlo predictions are compared with the results from the unfolding procedure shown in Table 3. The error bars represent the sum in quadrature of systematic and statistical errors. 
LIGHT MODEL
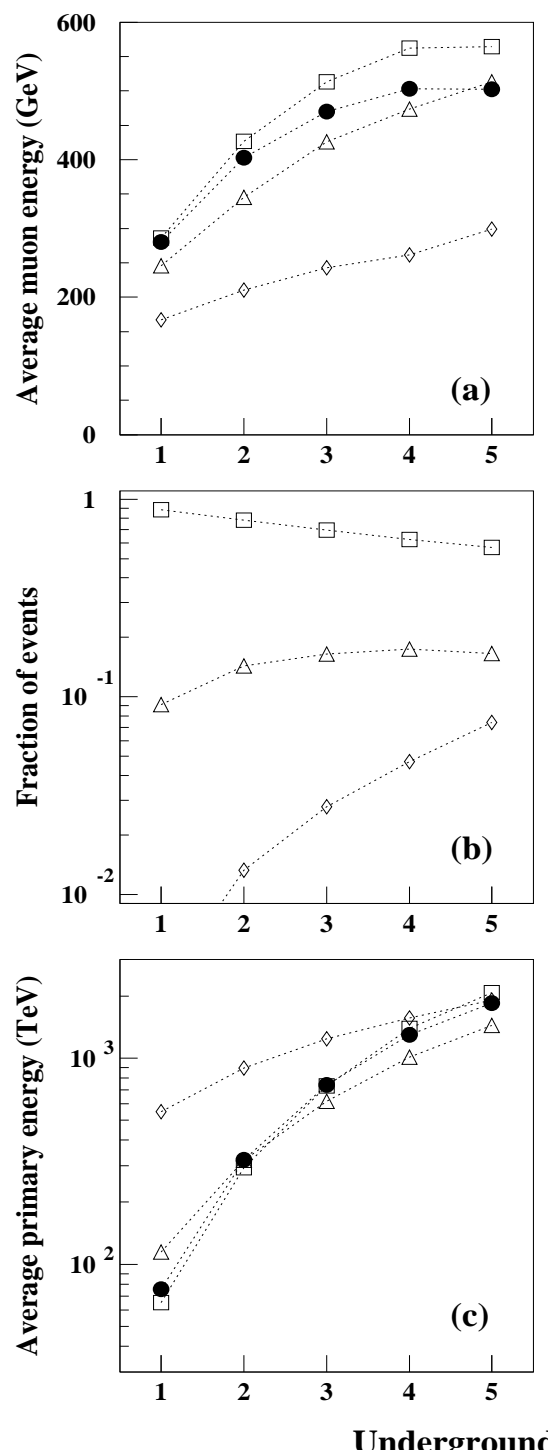

HEAVY MODEL
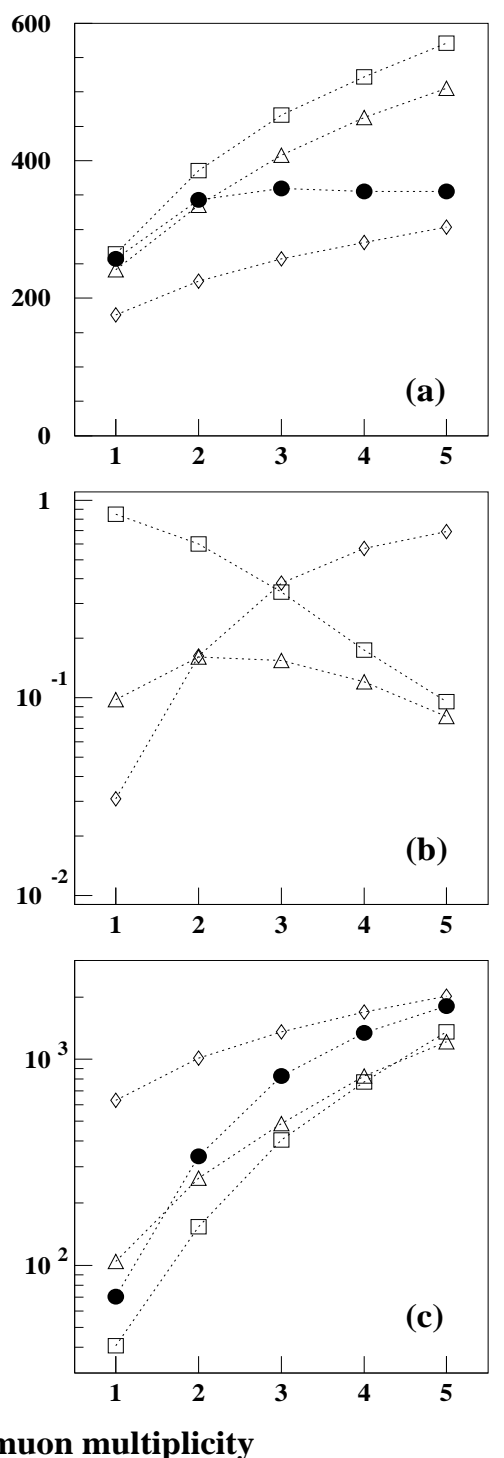

Figure 8. Physical quantities related to the underground muons produced by the main components of the primary cosmic rays $(\square=p, \Delta=H e, \diamond=F e, \bullet=$ all particles) plotted as a function of the muon multiplicity: a) average energies of underground muons; b) fraction of underground muons; c) average energies of the parent cosmic rays. Light (left panels) and heavy (right panels) composition models were separately considered. 

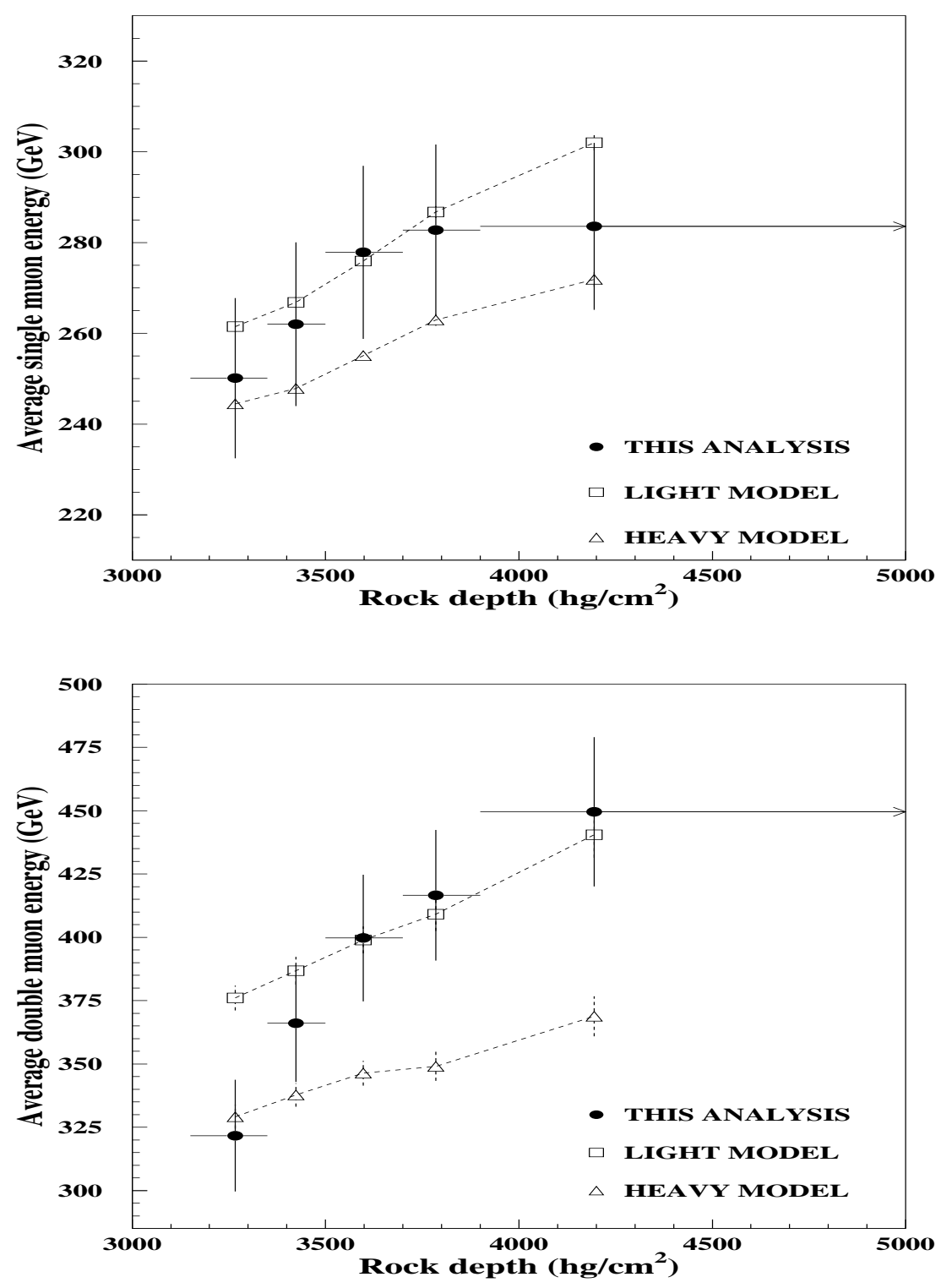

Figure 9. Average energies of single and double muons as a function of the rock depth. Statistical and systematic errors have been added in quadrature. The horizontal bars represent the width of the $h$ bins, while the central value of each bin corresponds to the average value of $h$ for that bin. The last bin extends up to $6500 \mathrm{hg} / \mathrm{cm}^{2}$. Results from the unfolding procedure are compared with the Monte Carlo predictions. 